\title{
CRITICAL AND MASSIVE PHASES IN SPIN CHAINS AND SPIN LADDERS
}

\author{
J. SólYOM \\ Research Institute for Solid State Physics, Hungarian Academy of Sciences \\ P.O. Box 49, 1525 Budapest, Hungary
}

Spin chains and spin ladders can have a variety of gapless (critical) or gapped (massive) phases depending on the length of the spin and the type of coupling. A brief review of the results on some simple models is given with emphasis on the generation of the Haldane gap in anisotropic spin ladders.

PACS numbers: $75.10 . \mathrm{Jm}$

\section{Introduction}

Spin chain models have been intensively studied ever since Bethe [1] has shown that the excitation spectrum of the spin-1/2 Heisenberg model can be calculated exactly by his famous Ansatz. Since this calculation gives a gapless spin liquid phase in the isotropic antiferromagnetic point, Haldane's conjecture $[2,3]$ that for integer spin models the spin liquid phase has a gap, was met with some scepticism. By now many analytical and numerical works support it.

The Haldane gap was first observed experimentally in $\mathrm{CsNiCl}_{3}$ [4]. Even more interestingly recently several classes of materials have been discovered in which two, three, etc. chains are coupled together to form spin ladders $[5,6]$. Here we will try to present some of our results, especially on the problem how the Haldane gap is generated in anisotropic spin ladders.

\section{Isotropic spin chain models}

The simplest isotropic model for the interaction of spins along a chain is the nearest neighbour Heisenberg model described by the Hamiltonian

$$
\mathcal{H}=J \sum_{i=1}^{N} \boldsymbol{S}_{i} \cdot \boldsymbol{S}_{i+1}
$$

where $i$ is the site index. We will mostly be interested in antiferromagnetic chains, which in our notation correspond to positive $J$. For $S>1 / 2$ higher powers of $\boldsymbol{S}_{i} \cdot \boldsymbol{S}_{i+1}$ may also appear. Assuming that only the nearest neighbour spins are coupled, the Hamiltonian can be written as

$$
\mathcal{H}=J \sum_{l=0}^{2 S} \sum_{i=1}^{N} f_{l} P_{l}\left(S_{i}+S_{i+1}\right),
$$


where $P_{l}\left(S_{i}+S_{i+1}\right)$ is the projection operator that projects to the subspace with $\left(S_{i}+S_{i+1}\right)^{2}=l(l+1)$. The usual antiferromagnetic Heisenberg model corresponds to the choice: $f_{l}=l(l+1)$.

For $S=1$, where bilinear and biquadratic terms may be present, the usual parametrization is

$$
\mathcal{H}=\sum_{i}\left[\cos \theta\left(\boldsymbol{S}_{i} \cdot \boldsymbol{S}_{i+1}\right)+\sin \theta\left(\boldsymbol{S}_{i} \cdot \boldsymbol{S}_{i+1}\right)^{2}\right] .
$$

On the antiferromagnetic side $(-\pi / 2 \leq \theta \leq \pi / 2)$ there are two special points, $\theta=-\pi / 4[7,8]$, and $\theta=\pi / 4[9,10]$, where the spectrum can be calculated exactly. In the first case the excitation spectrum is analogous to that of the antiferromagnetic spin- $1 / 2$ model, while in the second case there are three branches of soft modes.

Between these two points, i.e., for $-\pi / 4<\theta<\pi / 4$ the system is in the massive Haldane phase. The ground state is non-degenerate or fourfold degenerate, depending on the boundary condition, and in any case the ground state is separated from the continuum of excitations by a finite gap. Although the Haldane phase is a spin-liquid phase, there exists a hidden order characterized by the string order parameter [11]:

$$
\mathcal{O}_{\alpha}=-\lim _{|i-j| \rightarrow \infty}\left\langle S_{i}^{\alpha} \exp \left(\sum_{k=i+1}^{j-1} S_{k}^{\alpha}\right) S_{j}^{\alpha}\right\rangle, \quad \alpha=x, y, z .
$$

In the range $\pi / 4 \leq \theta \leq \pi / 2$ the system remains gapless [12] with three branches of soft modes. In the range $-3 \pi / 4 \leq \theta \leq-\pi / \dot{4}$ on the other hand the spectrum is again massive [13], and the ground state is doubly degenerate. The existence of a further new phase in the neighbourhood of $\theta=-3 \pi / 4$ has been proposed $[14,15]$, but so far it could not be detected in the numerical calculations [16].

Gap can be generated in isotropic antiferromagnetic spin- $1 / 2$ models as well, if the exchange between second neighbours is also important. Using lower case $s$ for spin- $1 / 2$ operators, the Hamiltonian is

$$
\mathcal{H}=J_{1} \sum_{i} s_{i} \cdot s_{i+1}+J_{2} \sum_{i} s_{i} \cdot s_{i+2} .
$$

At the special $J_{2} / J_{1}=1 / 2$ Majumdar-Ghosh point [17] the Hamiltonian can be written in terms of a spin-projection operator that projects the sum of three neighbouring spin operators to the maximal $l=3 / 2$ subspace

$$
\mathcal{H}=\frac{1}{4} J_{1} \sum_{i} P_{3 / 2}\left(s_{i-1}+s_{i}+s_{i+1}\right)-\frac{3}{4} J_{1} N
$$

This allows to construct the ground state as a dimerized first neighbour valence bond solid, and therefore it has a finite gap. In fact, the gap appears for $J_{2} / J_{1}>$ $\left(J_{2} / J_{1}\right)_{\mathrm{c}}=0.241$ already.

An analogous special point exists in the $S=1$ bilinear-biquadratic model

$$
\mathcal{H}=\sum_{i}\left[\left(\boldsymbol{S}_{i} \cdot \boldsymbol{S}_{i+1}\right)+\frac{1}{3}\left(\boldsymbol{S}_{i} \cdot \boldsymbol{S}_{i+1}\right)^{2}\right] \text {. }
$$


Apart from a constant term the coupling between neighbouring spins is proportional to the projection operator $P_{2}\left(S_{i}+S_{i+1}\right)$, and therefore the exact ground state wave function can be constructed $[18,19]$. It is a translationally invariant valence bond solid with nearest neighbour valence bonds only. The Haldane gap thus corresponds to the binding energy of a valence bond. Using this technique in fact a large number of other massive spin-1 models can be constructed [20].

\section{Isotropic spin ladders}

Spin ladder models are obtained by coupling two or more spin chains in an appropriate manner as shown in Fig. 1.

Introducing a new index $l$ that denotes the leg of the ladder, isotropic spin-1/2 ladders can be described by the Hamiltonian

$$
\mathcal{H}=\mathcal{H}_{0}+\mathcal{H}_{1}=\sum_{l} \mathcal{H}_{l}+\frac{1}{2} \sum_{l \neq l^{\prime}} \overrightarrow{\mathcal{H}}_{l^{\prime}}
$$

where the intrachain coupling is that of the usual Heisenberg chain,

$$
\mathcal{H}_{l}=J \sum_{i} s_{i, l} \cdot s_{i+1, l}
$$

while the interchain coupling acts between spins on the same rung,

$$
\mathcal{H}_{l^{\prime}}=J_{\perp} \sum_{i} s_{i, l} \cdot s_{i, l^{\prime}}
$$

When $J_{\perp}$ is ferromagnetic, the spins on the same rung add up to a large effective spin. Depending on the number of legs this effective spin is either integer (for even number of legs) or half-odd integer (for odd number of legs). Therefore a spin ladder with even number of legs in which antiferromagnetic spin chains are ferromagnetically coupled, will have a Haldane gap.

The Majumdar-Ghosh model can also be considered as a special coupled chain model. If the spins on even and odd sites are arranged in separate chains, as shown in Fig. 2, the first and second neighbour couplings give a ladder-like structure. If both of these couplings are antiferromagnetic, the frustration of the spins on triangles gives rise to the finite gap.

Similar diagonally coupled ladders but with Haldane-like gap can be obtained from the composite-spin representation of spin- $S$ chains, if $S_{i}$ is represented as the

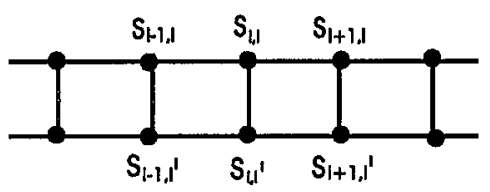

Fig. 1

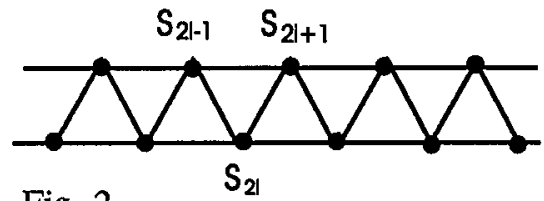

Fig. 2

Fig. 1. Intrachain and interchain coupling in a two-leg spin ladder.

Fig. 2. Ladder representation of the spin chain with first and second neighbour couplings. 
sum of $p=2 S$ spin- $1 / 2$ operators

$$
S_{i}=s_{i, 1}+s_{i, 2}+\ldots+s_{i, p} \text {. }
$$

Inserting this into Eq. (16), we get

$$
\mathcal{H}=\mathcal{H}_{0}+\mathcal{H}_{2}=\sum_{l=1}^{p} \mathcal{H}_{l}+\frac{1}{2} \sum_{l \neq l^{\prime}} \mathcal{H}_{l^{\prime}}
$$

where $\mathcal{H}_{l}$ has the same form as in Eq. (9), while the interchain coupling is

$$
\mathcal{H}_{l l^{\prime}}=J_{\perp} \sum_{i}\left(s_{i, l} \cdot s_{i+1, l^{\prime}}+s_{i, l^{\prime}} \cdot s_{i+1, l}\right) \text {. }
$$

This can be considered as the Hamiltonian of a spin ladder, in which the interchain coupling acts between spins on neighbouring rungs, as shown in Fig. 3.

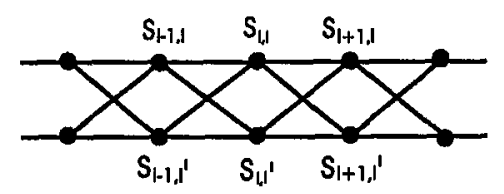

Fig. 3. Intrachain and interchain couplings in a diagonal ladder.

In this representation the Haldane phase is expected for antiferromagnetic diagonal interchain coupling, since this corresponds to the antiferromagnetic spin- $S$ chain, while in the usual ladder the Haldane phase is expected to occur for ferromagnetic interchain coupling. It has been shown [21] that the antiferromagnetic diagonal ladder is in the same universality class as the usual antiferromagnetic ladder, thus in the usual two-leg ladder the Haldane gap is generated for both signs of the interchain coupling.

Even more complicated couplings are obtained if the composite-spin representation is used for the $S=1$ bilinear-biquadratic model of Eq. (3). Beside the intrachain, the usual ladder-like and diagonal interchain couplings a new type of plaquette coupling appears, which in the isotropic case has the form

$$
\mathcal{H}_{3}=\sum_{i}\left[\left(s_{i, 1} \cdot s_{i+1,1}\right)\left(s_{i, 2} \cdot s_{i+1,2}\right)+\left(s_{i, 1} \cdot s_{i+1,2}\right)\left(s_{i, 2} \cdot s_{i+1,1}\right)\right] .
$$

In the composite-spin representation of the spin- 1 chain there is a special relationship between the strength of the various terms. In a ladder the couplings are independent. A general isotropic two-leg ladder could be described by the Hamiltonian

$$
\mathcal{H}=\lambda_{0} \mathcal{H}_{0}+\lambda_{1} \mathcal{H}_{1}+\lambda_{2} \mathcal{H}_{2}+\lambda_{3} \mathcal{H}_{3}
$$

with arbitrary couplings $\lambda_{i}$.

\section{Haldane phase in the diagonal ladder}

Earlier numerical calculations have already established [22,23] that the Haldane gap is generated in the two-leg ladder for arbitrary small $\lambda_{1}$ irrespective 
of the sign. We have performed numerical calculations [24] on a model that included $\mathcal{H}_{2}$ and $\mathcal{H}_{3}$ as well, using the density matrix renormalization group (DMRG) method [25, 26].

It was shown that like $\lambda_{1}$, the diagonal coupling also generates a Haldane gap for any $\lambda_{2} \neq 0$. There is, however, one feature that distinguishes the phases obtained for positive or negative couplings. Since it is convenient to use open boundary condition in the DMRG procedure, a fourfold degenerate ground state should be found due to the end spins. For $\lambda_{1}<0$ or $\lambda_{2}>0$ this is in fact the case. For $\lambda_{1}>0$ or $\lambda_{2}<0$, on the other hand, we find a non-degenerate ground state. This can be explained if we take into account that the usual and diagonal ladder can be transformed into each other by a shift of the spins on one of the legs, and that corresponds to cutting off the unpaired end spins that cause the extra degeneracy.

The plaquett coupling $\lambda_{3}$ alone will also generate Haldane gap, but its competition with the other couplings will lead to new phases. One finds a massive phase, where the ground state is doubly degenerate, which is related to the dimerized phase of the bilinear-biquadratic model, and surprisingly an extended critical phase, too.

\section{Anisotropic spin chains and spin ladders}

In many cases the exchange coupling between the spins is anisotropic. For uniaxial anisotropy the nearest neighbour Heisenberg Hamiltonian becomes

$$
\mathcal{H}=J \sum_{i}\left[\left(S_{i}^{x} S_{i+1}^{x}+S_{i}^{y} S_{i+1}^{y}\right)+\Delta S_{i}^{z} S_{i+1}^{z}\right] .
$$

The ferromagnetic ground state is stable for $\Delta \leq-1$. For half-odd integer spin values Néel-type antiferromagnetic order appears for $\Delta>1$, while in the range $-1<\Delta \leq 1$ the system is in a spin-liquid phase with gapless excitations. For integer spin values the Haldane phase appears between the gapless spin-liquid phase and the antiferromagnetic phase in a more or less narrow range around $\Delta=1$.

The anisotropic diagonal spin-1/2 ladders can be obtained analogously to the isotropic situation, using the composite-spin representation of the anisotropic spin- $S$ chain. The Hamiltonian will appear again as the sum of intrachain and interchain terms, with the slight modification that now

$$
\mathcal{H}_{l}=J \sum_{i}\left[\left(s_{i, l}^{x} s_{i+1, l}^{x}+s_{i, l}^{y} s_{i+1, l}^{y}\right)+\Delta s_{i, l}^{z} s_{i+1, l}^{z}\right],
$$

and

$$
\begin{aligned}
\mathcal{H}_{l^{\prime}} & =J_{\perp} \sum_{i}\left[\left(s_{i, l}^{x} s_{i+1, l^{\prime}}^{x}+s_{i, l^{\prime}}^{x} s_{i+1, l}^{x}+s_{i, l}^{y} s_{i+1, l^{\prime}}^{y}+s_{i, l}^{y} s_{i+1, l}^{y}\right)\right. \\
& \left.+\Delta_{\perp}\left(s_{i, l}^{z} s_{i+1, l^{\prime}}^{z}+s_{i, l^{\prime}}^{z} s_{i+1, l}^{z}\right)\right] .
\end{aligned}
$$

When $J_{\perp}=J$ and $\Delta_{\perp}=\Delta$, the diagonal ladder should have the properties of the spin- $S$ chain, i.e., for the two-leg ladder the gapped Haldane phase should extend from $\Delta=0$ to $\Delta=1.18$, while for the four-leg ladder it is limited to a 

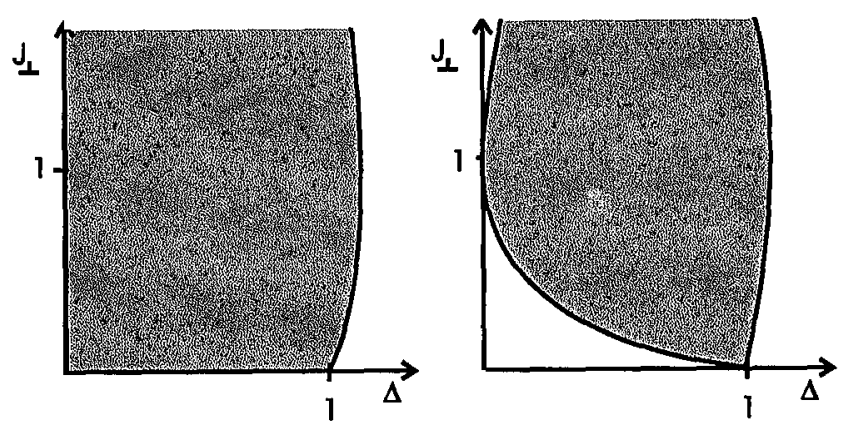

Fig. 4. Possible phase diagram of the two-leg diagonal ladder.
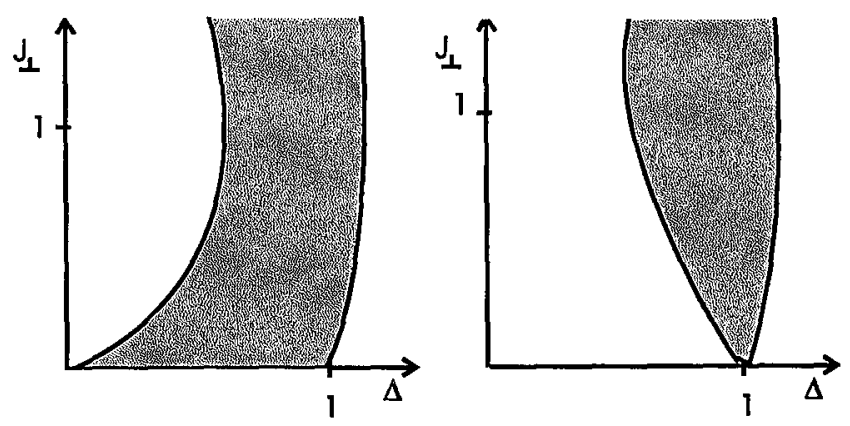

Fig. 5. Possible phase diagram of the four-leg diagonal ladder.

really narrow range around $\Delta=1$. One can ask the question how the Haldane gap appears when two or four anisotropic $S=1 / 2$ chains are coupled together. There are two rather natural expectations as shown in Fig. 4 and Fig. 5, respectively.

Either the interchain coupling is relevant, and the gap is generated for arbitrary small $J_{\perp}$, or it is irrelevant, and a finite coupling is needed to generate the gap.

We have performed numerical calculations [27] to decide which scenario is valid, but due to the smallness of the gap we were unable to draw definite conclusions. We have therefore done an analytic study [28] of the properties of spin ladders using the bosonized version of the model.

\section{Bosonized form of the two-leg ladder model}

The spin-1/2 operators can be transformed to spinless fermions using the Jordan-Wigner transformation and then the one-dimensional fermion model is bosonized. This corresponds to introducing $\Phi_{l}$ and $\Theta_{l}$ continuum phase fields and their adjoints for each chain. Using the standard procedure as given e.g., in Ref. [29], a single anisotropic spin-1/2 Heisenberg chain in its critical region is transformed into a simple harmonic Hamiltonian

$$
\mathcal{H}_{l}=\frac{u}{2} \int \mathrm{d} x\left[K \Pi_{l}^{2}+\frac{1}{K}\left(\partial_{x} \Phi_{l}\right)^{2}\right]
$$


where

$$
K=\frac{\pi}{2(\pi-\arccos \Delta)}, \quad u=J \frac{\pi \sqrt{1-\Delta^{2}}}{2 \arccos \Delta} .
$$

For coupled chains the $\Phi_{l}$ and $\Theta_{l}$ fields of the individual chains will be coupled. Using the bosonized form for the spin operators, we get

$$
\begin{aligned}
\mathcal{H}_{l, l^{\prime}} & =\int \frac{\mathrm{d} x}{(2 \pi a)^{2}}\left\{g_{1} \cos \left[\sqrt{4 \pi}\left(\Phi_{l}+\Phi_{l^{\prime}}\right)\right]\right. \\
& \left.+g_{2} \cos \left[\sqrt{4 \pi}\left(\Phi_{l}-\Phi_{l^{\prime}}\right)\right]+g_{3} \cos \left[\sqrt{\pi}\left(\Theta_{l}-\Theta_{l^{\prime}}\right)\right]\right\} \\
& +\frac{2 J_{\perp} \Delta_{\perp}}{\pi} \int \mathrm{d} x \partial_{x} \Phi_{l} \partial_{x} \Phi_{l^{\prime}} \\
& +g_{4} \int \frac{\mathrm{d} x}{(2 \pi a)^{2}} \cos \left[\sqrt{\pi}\left(\Theta_{l}-\Theta_{l^{\prime}}\right)\right] \cos \left[\sqrt{4 \pi}\left(\Phi_{l}+\Phi_{l^{\prime}}\right)\right] \\
& +g_{5} \int \frac{\mathrm{d} x}{(2 \pi a)^{2}} \cos \left[\sqrt{\pi}\left(\Theta_{l}-\Theta_{l^{\prime}}\right)\right] \cos \left[\sqrt{4 \pi}\left(\Phi_{l}-\Phi_{l^{\prime}}\right)\right]
\end{aligned}
$$

where $g_{1}=4 J_{\perp} \Delta_{\perp}, g_{2}=-4 J_{\perp} \Delta_{\perp}, g_{3}=-4 \pi J_{\perp}, g_{4}=g_{5}=2 \pi J_{\perp}$.

For the two-leg ladder it is useful to define symmetric and antisymmetric combinations of the fields

$$
\Phi_{\mathrm{s}}=\frac{1}{\sqrt{2}}\left(\Phi_{1}+\Phi_{2}\right), \quad \Phi_{\mathrm{a}}=\frac{1}{\sqrt{2}}\left(\Phi_{1}-\Phi_{2}\right) .
$$

In terms of these fields the Hamiltonian of the diagonal ladder has the form

$$
\begin{aligned}
\mathcal{H}= & \frac{u_{\mathrm{s}}}{2} \int \mathrm{d} x\left[K_{\mathrm{s}} \Pi_{\mathrm{s}}^{2}+\frac{1}{K_{\mathrm{s}}}\left(\partial_{x} \Phi_{\mathrm{s}}\right)^{2}\right]+g_{1} \int \frac{\mathrm{d} x}{(2 \pi a)^{2}} \cos \left(\sqrt{8 \pi} \Phi_{\mathrm{s}}\right) \\
& +\frac{u_{\mathrm{a}}}{2} \int \mathrm{d} x\left[K_{\mathrm{a}} \Pi_{\mathrm{a}}^{2}+\frac{1}{K_{\mathrm{a}}}\left(\partial_{x} \Phi_{\mathrm{a}}\right)^{2}\right] \\
& +\int \frac{\mathrm{d} x}{(2 \pi a)^{2}}\left[g_{2} \cos \left(\sqrt{8 \pi} \Phi_{\mathrm{a}}\right)+g_{3} \cos \left(\sqrt{2 \pi} \Theta_{\mathrm{a}}\right)\right] \\
& +g_{4} \int \frac{\mathrm{d} x}{(2 \pi a)^{2}} \cos \left(\sqrt{2 \pi} \Theta_{\mathrm{a}}\right) \cos \left(\sqrt{8 \pi} \Phi_{\mathrm{s}}\right) \\
& +g_{5} \int \frac{\mathrm{d} x}{(2 \pi a)^{2}} \cos \left(\sqrt{2 \pi} \Theta_{\mathrm{a}}\right) \cos \left(\sqrt{8 \pi} \Phi_{\mathrm{a}}\right)
\end{aligned}
$$

where

$$
\begin{array}{ll}
u_{\mathrm{s}}=u\left(1+\frac{2 K J_{\perp} \Delta_{\perp}}{u \pi}\right)^{1 / 2}, & K_{\mathrm{s}}=K\left(1+\frac{2 K J_{\perp} \Delta_{\perp}}{u \pi}\right)^{-1 / 2}, \\
u_{\mathrm{a}}=u\left(1-\frac{2 K J_{\perp} \Delta_{\perp}}{u \pi}\right)^{1 / 2}, & K_{\mathrm{a}}=K\left(1-\frac{2 K J_{\perp} \Delta_{\perp}}{u \pi}\right)^{-1 / 2} .
\end{array}
$$

Analyzing the scaling dimension of the various terms it turns out that both the symmetric and the antisymmetric modes will get gapped in the entire $0<\Delta \leq 1$ range when the interchain coupling is switched on. 


\section{Renormalization group treatment of the four-leg ladder}

For four-leg diagonal ladders similarly one can introduce a symmetric and three antisymmetric combinations

$$
\begin{array}{ll}
\Phi_{\mathrm{s}}=\frac{1}{2}\left(\Phi_{1}+\Phi_{2}+\Phi_{3}+\Phi_{4}\right), & \Phi_{\mathrm{a} 1}=\frac{1}{2} 7\left(\Phi_{1}+\Phi_{2}-\Phi_{3}-\Phi_{4}\right), \\
\Phi_{\mathrm{a} 2}=\frac{1}{2}\left(\Phi_{1}-\Phi_{2}-\Phi_{3}+\Phi_{4}\right), & \Phi_{\mathrm{a} 3}=\frac{1}{2}\left(\Phi_{1}-\Phi_{2}+\Phi_{3}-\Phi_{4}\right) .
\end{array}
$$

The parameters appearing in the harmonic part of the Hamiltonian are

$$
\begin{array}{ll}
u_{\mathrm{s}}=u\left(1+\frac{6 K J_{\perp}^{z}}{u \pi}\right)^{1 / 2}, & K_{\mathrm{s}}=K\left(1+\frac{6 K J_{\perp}^{z}}{u \pi}\right)^{-1 / 2}, \\
u_{\mathrm{a}}=u\left(1-\frac{2 K J_{\perp}^{z}}{u \pi}\right)^{1 / 2}, & K_{\mathrm{a}}=K\left(1-\frac{2 K J_{\perp}^{z}}{u \pi}\right)^{-1 / 2} .
\end{array}
$$

Without writing the full Hamiltonian we give just a few typical terms arising from the interchain coupling

$$
\begin{aligned}
& 2 g_{1} \int \frac{\mathrm{d} x}{(2 \pi a)^{2}} \cos \left(\sqrt{4 \pi} \Phi_{\mathrm{a} 1}\right) \cos \left(\sqrt{4 \pi} \Phi_{\mathrm{s}}\right) \\
& \quad+2 g_{2} \int \frac{\mathrm{d} x}{(2 \pi a)^{2}} \cos \left(\sqrt{4 \pi} \Phi_{\mathrm{a} 2}\right) \cos \left(\sqrt{4 \pi} \Phi_{\mathrm{a} 1}\right) \\
& \quad+2 g_{3} \int \frac{\mathrm{d} x}{(2 \pi a)^{2}} \cos \left(\sqrt{\pi} \Theta_{\mathrm{a} 2}\right) \cos \left(\sqrt{\pi} \Theta_{\mathrm{a} 1}\right) \\
& \quad+g_{4} \int \frac{\mathrm{d} x}{(2 \pi a)^{2}}\left\{\cos \left[\sqrt{\pi}\left(\Theta_{\mathrm{a} 3}+\Theta_{\mathrm{a} 2}\right)\right] \cos \left[\sqrt{4 \pi}\left(\Phi_{\mathrm{s}}+\Phi_{\mathrm{a} 1}\right)\right]\right. \\
& \left.\quad+\cos \left[\sqrt{\pi}\left(\Theta_{\mathrm{a} 3}-\Theta_{\mathrm{a} 2}\right)\right] \cos \left[\sqrt{4 \pi}\left(\Phi_{\mathrm{s}}-\Phi_{\mathrm{a} 1}\right)\right]\right\} \\
& \quad+g_{5} \int \frac{\mathrm{d} x}{(2 \pi a)^{2}}\left\{\cos \left[\sqrt{\pi}\left(\Theta_{\mathrm{a} 3}+\Theta_{\mathrm{a} 2}\right)\right] \cos \left[\sqrt{4 \pi}\left(\Phi_{\mathrm{a} 3}+\Phi_{\mathrm{a} 2}\right)\right]\right. \\
& \left.\quad+\cos \left[\sqrt{\pi}\left(\Theta_{\mathrm{a} 3}-\Theta_{\mathrm{a} 2}\right)\right] \cos \left[\sqrt{4 \pi}\left(\Phi_{\mathrm{a} 3}-\Phi_{\mathrm{a} 2}\right)\right]\right\} .
\end{aligned}
$$

As can be seen, there are terms that couple the symmetric mode to the antisymmetric modes, and others that only couple the antisymmetric modes among
themselves.

Since there are many terms than can compete we derived renormalization group equations for the couplings,

$$
\begin{aligned}
& \frac{\mathrm{d} g_{1}}{\mathrm{~d} l}=\left[2-\left(K_{\mathrm{s}}+K_{\mathrm{a}}\right)\right] g_{1}, \quad \frac{\mathrm{d} g_{2}}{\mathrm{~d} l}=\left(2-2 K_{\mathrm{a}}\right) g_{2}, \\
& \frac{\mathrm{d} g_{3}}{\mathrm{~d} l}=\left(2-\frac{1}{2 K_{\mathrm{a}}}\right) g_{3}, \quad \frac{\mathrm{d} g_{4}}{\mathrm{~d} l}=\left[2-\left(K_{\mathrm{s}}+K_{\mathrm{a}}+\frac{1}{2 K_{\mathrm{a}}}\right)\right] g_{4},
\end{aligned}
$$




$$
\begin{aligned}
\frac{\mathrm{d} K_{\mathrm{s}}}{\mathrm{d} l} & =-6 g_{1}^{2}\left(\frac{K_{\mathrm{s}}}{4 \pi u_{\mathrm{s}}}\right)^{2}-3 g_{4}^{2}\left(\frac{K_{\mathrm{s}}}{4 \pi u_{\mathrm{s}}}\right)^{2} \\
\frac{\mathrm{d} K_{\mathrm{a}}}{\mathrm{d} l} & =-2 g_{1}^{2}\left(\frac{K_{\mathrm{a}}}{4 \pi u_{\mathrm{a}}}\right)^{2}-4 g_{2}^{2}\left(\frac{K_{\mathrm{a}}}{4 \pi u_{\mathrm{a}}}\right)^{2}+g_{3}^{2}\left(\frac{1}{4 \pi u_{\mathrm{a}}}\right)^{2} \\
& -g_{4}^{2}\left(\frac{K_{\mathrm{a}}}{4 \pi u_{\mathrm{a}}}\right)^{2}+\frac{1}{2} g_{4}^{2}\left(\frac{1}{4 \pi u_{\mathrm{a}}}\right)^{2},
\end{aligned}
$$

and solved them numerically. It turned out that in this case the antisymmetric modes become massive, but the symmetric mode remains gapless.

\section{Conclusion}

The Haldane gap is a generic feature of isotropic antiferromagnetic integer spin magnetic chains or even-leg spin ladders. Gapless or dimerized phases may appear if anisotropy or higher order couplings are taken into account. Such higher order (plaquette) couplings can be obtained if the ladder model is derived from the composite-spin representation of a spin- $S$ chain.

The effect of anisotropy is even more surprising. Coupling two or four gapless anisotropic spin- $1 / 2$ chains into ladder, completely different scenarios are found. In the two-leg ladder an arbitrarily small interchain coupling will generate a Haldane gap in the entire range $0<\Delta \leq 1$, thus realizing the scenario shown in Fig. 1, while when four spin- $1 / 2$ chains are coupled into a four-leg ladder there always remain a gapless mode, and only a finite value of the interchain coupling can generate a Haldane gap in a narrow range of anisotropy.

\section{Acknowledgments}

The author is grateful to G. Fáth, E.H. Kim, and Ö. Legeza for the collaborations which led to most of our results presented in this paper. I acknowledge the partial support of the Hungarian Research Fund (OTKA) grant No. 30173.

\section{References}

[1] H. Bethe, Z. Phys. 71, 205 (1931).

[2] F.D.M. Haldane, Phys. Rev. Lett. 50, 1153 (1983).

[3] F.D.M. Haldane, Phys. Lett. A 93, 464 (1983).

[4] W.J.L. Buyers, R.M. Morra, R.L. Armstrong, M.J. Hogan, P. Gerlach, K. Hirakawa, Phys. Rev. Lett. 56, 371 (1986).

[5] D.C. Johnston, J.W. Johnson, D.P. Goshorn, A.C. Jacobson, Phys. Rev. B 35, 219 (1987).

[6] Z. Hiroi, M. Azuma, M. Takano, Y. Bando, J. Solid State Chem. 95, 230 (1991).

[7] L.A. Takhtajan, Phys. Lett. A 87, 479 (1982).

[8] H.M. Babujian, Phys. Lett. A 90, 479 (1982).

[9] C.K. Lai, J. Math. Phys. 15, 1675 (1974). 
[10] B. Sutherland, Phys. Rev. B 12, 3795 (1975).

[11] M. den Nijs, K. Rommelse, Phys. Rev. B 40, 4709 (1989).

[12] G. Fáth, J. Sólyom, Phys. Rev. $B$ 44, 11836 (1991).

[13] I. Affleck, Nucl. Phys. B 265 [FS 15], 409 (1986).

[14] A.V. Chubukov, J. Phys., Condens. Matter 2, 1593 (1990).

[15] A.V. Chubukov, Phys. Rev. B 43, 3337 (1991).

[16] G. Fáth, J. Sólyom, Phys. Rev. B 51, 3620 (1995).

[17] C.K. Majumdar, D.K. Ghosh, J. Math. Phys. 10, 1388 (1969).

[18] I. Affleck, T. Kennedy, E.H. Lieb, H. Tasaki, Phys. Rev. Lett. 59, 799 (1987).

[19] I. Affleck, T. Kennedy, E.H. Lieb, H. Tasaki, Commun. Math. Phys. 115, 477 (1988).

[20] A. Klümper, A. Schadschneider, J. Zittartz, Europhys. Lett. 24, 293 (1993).

[21] S.R. White, Phys. Rev. B 53, 52 (1996).

[22] S. Takada, H. Watanabe, J. Phys. Soc. Jap. 61, 39 (1992).

[23] T. Barnes, E. Dagotto, J. Riera, E.S. Swanson, Phys. Rev. B 47, 3196 (1993).

[24] Ö. Legeza, G. Fáth, J. Sólyom, Phys. Rev. B 55, 291 (1997).

[25] S.R. White, Phys. Rev. Lett. 69, 2863 (1992).

[26] S.R. White, Phys. Rev. B 48, 10345 (1993).

[27] Ö. Legeza, J. Sólyom, Phys. Rev. B 56, 14449 (1997).

[28] E.H. Kim, J. Sólyom, Phys. Rev. B, to be published.

[29] A.M. Tsvelik, Quantum Field Theory in Condensed Matter Physics, Cambridge University Press, Cambridge 1995. 